\title{
Hidroterapia no equilíbrio dinâmico e nas atividades de vida diária de pacientes com neurotoxoplasmose associada à Síndrome da Imunodeficiência Adquirida
}

\author{
Hydrotherapy on dynamic balance and in activities of daily living in patients with \\ neurotoxoplasmosis associated with the Acquired Immune Deficiency Syndrome
}

Larissa de Salgado de Oliveira ${ }^{1,2,3}$, Elciane Souza de Medeiros², Helber Thiago de Assunção Machado²,

Anderson Antunes da Costa Moraes ${ }^{3}$, Ediléa Monteiro de Oliveira ${ }^{3,4}$, Rodrigo Santiago Barbosa Rocha ${ }^{2,3}$

'Departamento de Intervenção Fisioterapêutica do Sistema Neuromuscular, Universidade Metodista de Piracicaba (UNIMEP) - Piracicaba (SP), Brasil.

${ }^{2}$ Curso de Fisioterapia, Universidade da Amazônia (UNAMA) - Belém (PA), Brasil.

${ }^{3}$ Curso de Fisioterapia, Universidade do Estado do Pará (UEPA) - Belém (PA), Brasil.

${ }^{4}$ Programa de Doutorado em Ciências da Reabilitação, Universidade Nove de Julho (UNINOVE) - São Paulo (SP), Brasil.

DOI: http://dx.doi.org/10.7322/abcshs.v4111.845

RESUMO

Introdução: Por comprometer as células de defesa do organismo, o Vírus da Imunodeficiência Humana torna o indivíduo vulnerável ao aparecimento de diversas doenças, entre elas a neurotoxoplasmose. Objetivo: Verificar a influência de um protocolo de hidroterapia no equilíbrio dinâmico e nas atividades de vida diária de pacientes com neurotoxoplasmose associada à Síndrome da Imunodeficiência Adquirida (SIDA). Métodos: Participaram 15 voluntários, três (20\%) do sexo feminino e 12 (80\%) do masculino, com média de idade de $37,44 \pm 5,5$ anos e diagnóstico de neurotoxoplasmose decorrente da SIDA, cadastrados na Unidade de Referência Especializada em Doenças Infectocontagiosas Parasitárias Especiais. Foram submetidos à avaliação do equilíbrio dinâmico pelo Índice de Marcha Dinâmico e à avaliação das atividades de vida diária pelo Índice de Barthel, pré- e pós-hidroterapia, em piscina à temperatura média de $35^{\circ} \mathrm{C}$, três vezes por semana em dias alternados, durante 50 minutos, totalizando oito semanas, ou seja, 24 sessões. Foi utilizado o teste de Shapiro-Wilk para análise de variâncias do Índice de Marcha Dinâmica e do Índice de Barthel, e o teste t de Student para as comparações pré e pós-tratamento e nível de significância de $\alpha=0.05$. Resultados: $\bigcirc$ equilíbrio dinâmico, considerando o escore total, apresentou significância estatística ( $p<0,0001)$, quando comparado pós-teste $(20,3 \pm 2,5)$ em relação ao pré-teste $(13,2 \pm 3,2)$. Quanto às atividades de vida diária, foi evidenciado valor estatisticamente significante $(p=0,049)$ no pós-teste $(98,8 \pm 2.2)$

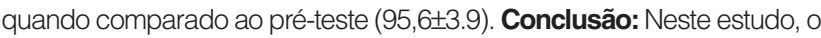
protocolo de hidroterapia melhorou o equilíbrio dinâmico e as atividades de vida diária de pacientes com neurotoxoplasmose associada à SIDA.

Palavras-chave: toxoplasmose cerebral; Síndrome da Imunodeficiência Adquirida; fisioterapia; hidroterapia.

\section{ABSTRACT}

Introduction: For compromising the defense cells of the body, the Human Immunodeficiency Virus makes the individual vulnerable to the emergence of various diseases, including the neurotoxoplasmosis. Objective: To determine the effect of a hydrotherapy protocol in dynamic balance and the activities of daily living of patients with cerebral toxoplasmosis associated with the Acquired Immuno Deficiency Syndrome (AIDS). Methods: 15 volunteers participated, three (20\%) were female and 12 of them $(80 \%)$ were male; mean age $37.44 \pm 5.5$ years, diagnosed with cerebral toxoplasmosis resulting from AIDS registered in Specialized Reference Unit on Infectious Diseases Parasitic Infectious Specials. Underwent evaluation of dynamic balance by the Dynamic Gait Index and the activities of daily living by Barthel Index, pre and post hydrotherapy, in the pool at an average temperature of $35^{\circ} \mathrm{C}, 3$ times per week on alternate days, lasting 50 minutes, a total of 8 weeks or 24 sessions. We used the Shapiro-Wilk test for analysis of the Dynamic Gait Index and Barthel Index variances, and T Student test for pre and post-treatment comparison and significance level of $\alpha=0.05$. Results: Total score of dynamic balance presented statistically significance $(p<0.0001)$, comparison post-test (20.3 \pm 2.5$)$ compared to pre-test (13.2 \pm 3.2$)$. As for the activities of daily living was evidenced statistically significant value $(p=0.049)$, and the post-test $(98.8 \pm 2.2)$ compared to pre-test (95.6 \pm 3.9$)$. Conclusion: In this study, hydrotherapy protocol improved the dynamic balance and the activities of daily living of patients with cerebral toxoplasmosis associated with AIDS.

Keywords: toxoplasmosis, cerebral; Acquired Immunodeficiency Syndrome; physical therapy specialty; hydrotherapy. 


\section{INTRODUÇÃO}

O Vírus da Imunodeficiência Humana (HIV) e a Síndrome da Imunodeficiência Adquirida (SIDA) constituem problemas de Saúde Pública, trazendo implicações para a sociedade nos âmbitos biopsicossocial, econômico e cultural ${ }^{1,2}$.

O HIV tem a capacidade de inserir seu material genético em células-alvo hospedeiras, principalmente os linfócitos T CD4, os quais constituem células de defesa do sistema imunológico humano. Após a infecção e ampla replicação, o vírus destrói várias células e, posteriormente, infecta outras. Essa infecção causa a SIDA, uma doença de espectro amplo, com curso clínico variável, progressiva supressão do sistema imunológico, indução a infecções oportunistas recorrentes incluindo desde um resfriado até infecções mais graves, alterações nutricionais, debilidade progressiva e morte ${ }^{3,4}$.

Entre as alterações mais comuns encontradas em portadores de SIDA, inclui-se a toxoplasmose do sistema nervoso central, que consiste em uma infecção oportunista muito comum em pacientes imunodeprimidos provocada por um protozoário chamado Toxoplasma gondii. Este, por sua vez, torna-se responsável por um grande número de lesões cerebrais focais, principalmente quando a contagem de linfócitos T CD4+é menor que 100 a 200 células $/ \mathrm{mm}^{3}$, atingindo cerca de $73 \%$ dos indivíduos HIV positivo ${ }^{5}$.

As manifestações clínicas observadas dependem da localização e da extensão da lesão, apresentando-se diversas vezes como uma encefalite difusa, meningoencefalite ou, mais comumente, como uma lesão tumoral com efeito de massa ${ }^{5,6}$. Outras alterações do quadro clínico caracterizam-se por mioclonias, tremores, convulsões, hemiparesia e ataxia, que podem afetar diretamente sua funcionalidade, fato que interfere na qualidade de vida ${ }^{2}$.

A disfunção motora causada pela hemiparesia refletirá na marcha, envolvendo déficit na velocidade, nas simetrias, no tempo e no comprimento do passo e da passada. Essas alterações não são apenas devidas à fraqueza muscular, mas também a anormalidades complexas do controle motor, do equilíbrio, do déficit sensorial e do tônus postural ${ }^{7,8}$.

Assim, a hidroterapia atua sobre essas limitações sensório-motoras buscando prevenir complicações secundárias, bem como manter ao máximo as capacidades funcionais ou potencializar a sua reinserção em funções anteriormente exercidas, em virtude dos benefícios adquiridos com as propriedades da água, como o alívio do peso durante os exercícios, a facilitação de movimentos, o relaxamento, o fortalecimento muscular e os treinos de equilíbrio e de atividades funcionais 9 .

Entretanto, um tratamento voltado para pacientes portadores da SIDA ainda é observado pela sociedade com muito preconceito, devido ao conhecimento equivocado quanto a determinadas formas de transmissão da doença. Somados aos dados estatísticos alarmantes, essa doença tem gerado impacto e pânico devastadores, além de despertar, difusamente, ansiedade, estigma, medo e rejeição ${ }^{10}$.

Assim sendo, torna-se de suma importância a discussão acerca da contribuição da hidroterapia sobre o equilíbrio dinâmico e a qualidade de vida em pacientes portadores da neurotoxoplasmose associada à SIDA. Dessa forma, o estudo objetivou verificar a influência de um protocolo de hidroterapia sobre o equilíbrio dinâmico e as atividades de vida diária de pacientes com neurotoxoplasmose associada à SIDA.

\section{MÉTODOS}

O estudo teve aprovação do Comitê de Ética em Pesquisa com Seres Humanos (CAAE no 420479/11) da Universidade da Amazônia (UNAMA) e assinatura do Termo de Consentimento Livre e Esclarecido (TCLE) pelos voluntários encaminhados da Unidade de Referência Especializada em Doenças Infectocontagiosas Parasitárias Especiais (URE-DIPE) para serem atendidos na Clínica Escola de Fisioterapia e Terapia Ocupacional (Fisioclínica) da UNAMA.

Foram inclusos na pesquisa 15 indivíduos com diagnóstico de neurotoxoplasmose como consequência da SIDA. Com relação à média de permanência do diagnóstico da SIDA, a amostra apresentou uma média de 8,22 $\pm 2,40$ anos e de diagnóstico de neurotoxoplasmose de 7,0 $\pm 1,9$ ano, com idades entre 24 e 44 anos, que não apresentaram infecções dermatológicas que impediam seu tratamento na água - mediante laudo médico e inspeção dermatológica -, que não estavam realizando tratamento fisioterapêutico, com estabilidade hemodinâmica, segundo as Diretrizes Brasileiras de Hipertensão Arterial (pressão sistólica $>90$ e $<140$; pressão diastólica $>50$ e <90), indivíduos que no Miniexame do Estado Mental apresentaram resposta acima de 23 pontos, levando em consideração a escolaridade, e que não apresentavam deformidades que interferissem nos resultados do estudo.

Foram excluídos os voluntários que apresentaram ulcerações cutâneas e/ou alergias relacionadas à pele, contagem de células $\mathrm{T}$ CD4 inferior a 200 células $/ \mathrm{mm}^{3}$ visualizadas em exame médico, insuficiência cardíaca, hipertensão arterial sistêmica não controlada, dispneia aos mínimos esforços, quaisquer tipos de sondas, déficit cognitivo acentuado e alterações visuais e auditivas que impedissem a compreensão dos comandos verbais e visualização das tarefas, que não tinham controle dos esfíncteres urinário e fecal, além daqueles com outras patologias neurológicas como acidente vascular encefálico, disfunções do aparelho vestibular e ataxia acentuada.

As variáveis-desfecho avaliadas foram o equilíbrio dinâmico por meio do Índice de Marcha Dinâmica e as atividades de vida diária verificadas pelo Índice de Barthel pré- e pós-intervenção. O Índice de Barthel consiste em um teste de mensuração do grau de independência em dez atividades de autocuidado: alimentação, banho, vestuário, higiene, dejeções, micção, sanitário, transferência, deambulação e escadas ${ }^{11}$. O Índice de Marcha Dinâmica, por sua vez, consiste em testes dinâmicos que incentivam estímulos vestibulares do transcorrer da marcha com obstáculos, degraus, entre outros. É composto por oito itens com quatro alternativas, que variam de 0 a 3 pontos, nas quais 0 indica grave 
comprometimento e 3 indica desempenho normal da função. A escala possui pontuação máxima de 24 pontos, tendo indicativo de risco de queda quando há um índice menor ou igual a 19 pontos $^{12}$.

Além disso, foi realizada a hidroterapia, individualmente, em piscina aquecida com temperatura média de $35^{\circ} \mathrm{C}$, por $50 \mathrm{mi}$ nutos, três vezes na semana durante oito semanas, totalizando 24 sessões. Foram utilizados equipamentos para o desenvolvimento do protocolo de tratamento, como aquatubos, tornozeleiras, luvas, bastões sinalizadores e steps.

O programa terapêutico foi composto por quatro etapas: aquecimento, alongamento, exercício aeróbio e desaquecimento.

$\mathrm{O}$ aquecimento ocorreu no primeiro momento da atividade e foi realizado por meio de cinco minutos de caminhada ao redor da piscina. Em seguida, foram realizados os alongamentos com tempo de manutenção de 30 segundos cada exercício: alongamento cruzado à frente para musculatura do trapézio, romboide, infraespinhal, redondo maior, redondo menor e deltoide posterior; puxada para trás por trás das costas para musculatura do peitoral menor, deltoide anterior e bíceps; puxada acima da cabeça para musculatura do grande dorsal, peitoral, redondo maior e tríceps; flexão lateral do tronco para musculatura do sacro-espinhal, quadrado lombar, oblíquo interno e externo; alongamento ativo dos isquiotibiais: musculatura dos isquiotibiais, glúteo máximo, gastrocnêmio e sóleo e alongamento de quadríceps.

As atividades aeróbicas eram realizadas por dois minutos cada exercício e compreenderam: caminhada de frente (utilizando os membros superiores realizando movimentos de estilo nado de peito), caminhada de deslocamento lateral (realizando movimentos de adução e abdução de ombro), caminhada de frente e volta de costa (utilizando membros superiores e realizando movimento alternado), caminhada contornando obstáculos (contornando bastões sinalizadores no fundo da piscina); step frontal (subir no step de frente e descer de costas) e step lateral (subir no step com a perna direita e descer com a esquerda e subir com a esquerda e descer com a direita); e simulação de bicicleta em flutuação com aquatubo (alternadamente, realizar movimentos de flexão e extensão de joelho e quadril, levando a perna a executar um movimento de bicicleta vertical). Ainda em flutuação supina com aquatubo, eram realizadas abdução e adução de quadril, flexão e extensão do quadril e batimento de pernas produzindo uma movimentação agitada. Em pé, eram realizadas dorsiflexão e flexão plantar de tornozelo. Além disso, havia o treino da largura do passo (com movimento de flexão e extensão de quadril em um membro, em apoio unipodal) e o batimento de pernas na posição pronada (apoiado na barra de apoio da piscina, alternadamente realizar a flexão e extensão de quadril produzindo uma movimentação agitada). A fase de desaquecimento teve duração de três minutos e foi realizada com o auxílio de equipamentos de flutuação.

Utilizou-se o teste de Shapiro-Wilk para normalidade dos dados e para a análise de variâncias do Índice de Marcha Dinâmica e do Índice de Barthel; o teste $t$ de Student foi usado para as comparações pré e pós-tratamento. Os dados foram processados no software BioEstat versão $5.2^{\circ}$, considerando um nível de significância de $\alpha=0.05$.

\section{RESULTADOS}

A amostra foi composta de 15 voluntários, três (20\%) do sexo feminino e 12 (80\%) do sexo masculino, com média de idade de $37,44 \pm 5,50$ anos. Com relação à média de permanência do diagnóstico da SIDA, a amostra apresentou uma média de 8,22 $\pm 2,40$ anos

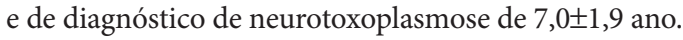

Os resultados referentes à Escala Índice de Marcha Dinâmico demonstraram que, após o tratamento com hidroterapia, houve aumento significativo nos valores médios do escore geral da escala, assim como das variáveis marcha em superfície plana, mudanças na velocidade da marcha, marcha com movimentos verticais de cabeça, marcha e rotação e andar ao redor de obstáculos $(\mathrm{p} \leq 0,05)$ conforme Tabela 1 .

$\mathrm{Na}$ Tabela 2 estão expressos os valores médios das atividades do Índice de Barthel pré- e pós-tratamento, respectivamente, na qual se observa melhora significativa nos itens alimentação, vestuário e deambulação.

Tabela 1: Valores médios \pm desvio padrão referente às oito atividades da Escala de Índice de Marcha Dinâmico e seu escore geral no pré- e no pós-tratamento na hidroterapia de indivíduos com neurotoxoplasmose associado à Síndrome da Imunodeficiência Adquirida

\begin{tabular}{|l|c|c|c|}
\hline Atividades & Antes & Depois & Valor p \\
\hline Marcha em superfície plana & $2,4 \pm 0,4$ & $3 \pm 0$ & $0,007^{*}$ \\
\hline Mudanças na velocidade da marcha & $2,5 \pm 0,4$ & $3 \pm 0$ & $0,003^{*}$ \\
\hline $\begin{array}{l}\text { Marcha com rotação } \\
\text { horizontal da cabeça }\end{array}$ & $2,8 \pm 0,4$ & $3 \pm 0$ & 0,070 \\
\hline $\begin{array}{l}\text { Marcha com } \\
\text { movimentos verticais de cabeça }\end{array}$ & $2,4 \pm 0,5$ & $2,8 \pm 0,4$ & $0,004^{*}$ \\
\hline Marcha e rotação & $2,0 \pm 0,7$ & $2,8 \pm 0,4$ & $0,021^{*}$ \\
\hline Passar por cima de um obstáculo & $3 \pm 0$ & $3 \pm 0$ & 0,102 \\
\hline Andar ao redor de obstáculos & $2,2 \pm 0,8$ & $2,6 \pm 0,8$ & $0,015^{*}$ \\
\hline Degraus & $2,8 \pm 0,5$ & $3 \pm 0$ & 0,063 \\
\hline Score total & $13,2 \pm 3,2$ & $20,3 \pm 2,5$ & $<0,0001^{*}$ \\
\hline
\end{tabular}

${ }^{*}$ Difere significativamente do pré-tratamento.

Tabela 2: Valores médios \pm desvio padrão do Índice de Barthel ( 0 a 100 pontos) de voluntários com neurotoxoplasmose associada à Síndrome da Imunodeficiência Adquirida antes e após as sessões de hidroterapia

\begin{tabular}{|l|c|c|c|}
\hline Atividades & Antes & Depois & Valor p \\
\hline Alimentação & $8,8 \pm 2,2$ & $9,4 \pm 1,6$ & $0,035^{*}$ \\
\hline Banho & $5 \pm 0$ & $5 \pm 0$ & 0,095 \\
\hline Vestuário & $8,8 \pm 2,2$ & $9,4 \pm 1,6$ & $0,035^{*}$ \\
\hline Higiene & $5 \pm 0$ & $5 \pm 0$ & 0,095 \\
\hline Dejeções & $10 \pm 0$ & $10 \pm 0$ & 0,457 \\
\hline Micção & $9,4 \pm 1,6$ & $9,4 \pm 1,6$ & 0,086 \\
\hline Sanitário & $10 \pm 0$ & $10 \pm 0$ & 0,457 \\
\hline Transferências & $14,4 \pm 1,6$ & $13,8 \pm 2,2$ & 0,065 \\
\hline Deambulação & $13,8 \pm 2,2$ & $15 \pm 0$ & $0,028^{*}$ \\
\hline Escadas & $10 \pm 0$ & $10 \pm 0$ & 0,457 \\
\hline Score total & $95,6 \pm 3,9$ & $98,8 \pm 2,2$ & 0,0497 \\
\hline
\end{tabular}

*Difere significativamente do pré-tratamento. 


\section{DISCUSSÃO}

O presente estudo destaca, entre seus achados sensório-motores, o comprometimento do equilíbrio corporal, desfecho importante nesta pesquisa, corroborando os estudos ${ }^{13,14}$ que também apontam tal déficit em portadores de neurotoxoplasmose associada à SIDA.

Vale ressaltar que o sistema de controle postural exige intrínseca interação entre aferências visual, vestibular e somatossensorial, centros neurais integradores, vias descendentes de controle e sistema musculoesquelético. Dentro desses sistemas existem dois parâmetros a se considerar, um envolvendo a orientação postural, ou seja, a manutenção da posição dos segmentos corporais em relação aos próprios segmentos e ao meio ambiente, e o outro, o equilíbrio postural, representado por relações entre as forças que agem sobre o corpo na busca do equilíbrio corporal durante as ações motoras para alcance da posição e orientação desejada ${ }^{8,15}$.

Dessa forma, a hipótese para o déficit de equilíbrio entre os voluntários do presente estudo deva-se aos comprometimentos sensoriais pela alteração do centro de gravidade em decorrência da não localização adequada do membro no espaço ${ }^{8,13,16}$.

Indivíduos hemiparéticos, como os presentes neste estudo, normalmente, têm déficits de equilíbrio devido à tendência de negligenciar o hemicorpo acometido por perderem parcialmente ou totalmente a percepção corporal, o que, por sua vez, desencadeia várias alterações, entre elas o aumento da base de suporte e a lateralização do centro de gravidade e tronco, fato que influencia diretamente na marcha e na postura ${ }^{17}$.

As dificuldades na realização de atividades funcionais decorrentes do déficit de equilíbrio nos indivíduos com neurotoxoplasmose e SIDA incluem instabilidade acentuada, distribuição assimétrica do peso corporal e diminuição da capacidade de manter o alinhamento da postura. Dessa maneira, o equilíbrio, a marcha e a mobilidade são alvos de intervenção no processo de reabilitação desses indivíduos ${ }^{4,6,13}$.

Devido ao protocolo do presente estudo envolver diversas atividades que trabalharam o déficit de equilíbrio dinâmico dos voluntários, foi possível observar pela aplicação da Escala Índice de Marcha Dinâmica uma melhora pós-intervenção. Possivelmente esses resultados ocorreram em virtude das alterações neurais e adaptações musculares em resposta aos exercícios propostos pelo protocolo de fisioterapia aquática aplicado ${ }^{18}$.

Tais resultados se assemelham ao estudo ${ }^{19}$ que utilizou um programa de exercícios em piscina com dez pacientes portadores de acidente vascular cerebral, os quais obtiveram como resposta melhora da performance da mobilidade funcional, do equilíbrio e da marcha.

O protocolo utilizado neste estudo enfatizou a estimulação do controle e ajuste do equilíbrio corporal dentro da água, com a realização de atividades motoras que abrangeram transferências posturais, marcha e utilização de degrau (step). Tais exercícios tiveram como objetivo dificultar a realização das tarefas para que se melhorasse a manutenção da simetria corporal, estimulando, dessa maneira, os sistemas responsáveis pelo equilíbrio do corpo $^{8,19}$.
Quando analisadas as atividades contidas no Índice de Marcha Dinâmica individualmente no pós-teste, foi observado que a marcha em superfície plana apresentou melhora no equilíbrio, o que pode ser explicado pela turbulência aquática utilizada para desenvolver habilidades de equilíbrio e coordenação. Durante a reavaliação da marcha com movimentos verticais da cabeça, foi verificada melhora significativa na execução de rotações uniformes da cabeça sem nenhuma mudança na marcha, o que pode ser explicado por uma melhora da força muscular e da reação dos músculos agonistas, facilitando, dessa forma, a mudança na direção dos movimentos, bem como uma maior coordenação destes ${ }^{20}$.

Quanto à análise da atividade marcha e rotação, o presente estudo obteve resultados satisfatórios, podendo ser explicados pela utilização de exercícios que aprimoraram as informações sensoriais do sistema vestibular, visual e somatossensorial, de modo a ativar a musculatura antigravitacional, responsável pela execução e manutenção de posturas estáticas e dinâmicas ${ }^{18}$. Os ganhos funcionais obtidos na tarefa após o programa de hidroterapia possivelmente estão relacionados à ação da turbulência, refração e pressão hidrostática, propriedades físicas presentes no meio aquático ${ }^{9,19}$.

Sobre a capacidade de mudar a velocidade da marcha de forma uniforme sem perda de equilíbrio, houve melhora. Entretanto, esses dados opõem-se aos resultados encontrados em outro estudo, no qual a terapêutica aquática não obteve êxito após a intervenção do protocolo utilizado no estudo ${ }^{21}$. Todavia, a melhora nessa atividade no presente estudo pode ser explicada pela ação da força de flutuação, que auxilia na realização dos movimentos na água, fazendo com que haja menos compensações na busca do equilíbrio e do movimento normal ${ }^{9}$.

$\mathrm{Na}$ atividade que consistia em andar ao redor de um obstáculo, os resultados indicam uma melhora significativa. A refração no meio aquático tem como característica gerar distorções na posição dos membros e da postura correta do indivíduo em ortostatismo, dessa forma, pode ter participado como estimulante dos mecanismos de compensação do equilíbrio no presente estudo ${ }^{9,22}$.

A segunda variável-desfecho relaciona-se às atividades de vida diária, que foram avaliadas por meio do Índice de Barthel, pré- e pós-intervenção. Trata-se de um instrumento amplamente utilizado com confiabilidade e validade muito consistentes ${ }^{23}$ e que, neste estudo, apresentou como resultado melhora da deambulação, vestuário e alimentação.

É importante salientar que as propriedades da água como a flutuação, a resistência e a pressão hidrostática auxiliam o fisioterapeuta na reabilitação, potencializando o fortalecimento muscular, a funcionalidade e o equilíbrio, proporcionando, ao mesmo tempo, um ambiente divertido, motivante e seguro ${ }^{9,12}$.

Pode-se inferir ainda que a propriedade de suporte que é conferida pela água possibilita a realização de atividades mais independentes por parte de indivíduos com alteração do equilíbrio corporal, proporcionando, assim, maior tempo de reação para se equilibrar ${ }^{20,22}$. 
Conclui-se, portanto, que, neste estudo, indivíduos portadores de neurotoxoplasmose associada à SIDA apresentaram melhora no equilíbrio dinâmico e nas atividades de vida diária pós-intervenção baseada em um protocolo de hidroterapia, tornando a fisioterapia aquática um importante meio de reabilitação funcional de distúrbios neurológicos.

\section{REFERÊNCIAS}

1. Silva J, Souza FMS, Lima MAS, Galvão JO, Pichelli AAWS. Quality of life in the context of HIV/AIDS: a comparative study with the general population. J Bras Doenças Sex Transm. 2013;25(2):88-92. http://dx.doi.org/10.5533/DST-2177-8264-201325207

2. Meirelles BHS, Silva DMGV, Vieira FMA, Souza SS, Coelho IZ, Batista R. Percepções da qualidade de vida de pessoas com hiv/ aids. Rev Rene. 2010;11(3):68-76.

3. Perrut JF, Santos EP, Oliveira BR, Carneiro ACLL, Oliveira GL, Ervilha Júnior, et al. Risco nutricional e sua associação com o tempo e desfecho da internação de pacientes com aids em um hospital de referência em infectologia de Belo Horizonte. Espaço Saúde. 2014;15(1):57-65.

4. Paula CC, Cabral IE, Souza IEO. Existential movement experienced by adolescents with acquired immunodeficiency syndrome: a phenomenological study. OBJN. 2013;12(1):33-48. http://dx.doi.org/10.5935/1676-4285.20133572

5. Santos IP, Martins FS, Rech G, Rosa BL, Borba Júnior AM. Déficit neurológico focal e confusão mental em paciente imunossuprimido. Rev Epidemiol Control Infect. 2013;3(3):108-9.

http://dx.doi.org/10.17058/reci.v3i3.3766

6. Soares MT, Pinheiro JBF, Freitas CHSM, Smith AZF, Moreira EFR Prevalência das coinfecções em pacientes notificados com Aids no Centro de Referência na Paraíba. Rev Bras Ciênc Saúde. 2014;18(Suppl 1):5-12. http://dx.doi.org/10.4034/RBCS.2014.18.s1.01

7. Alferes DC, Ribeiro MA. Toxoplasmose cerebral: um caso clínico. Rev Científica Acad Força Aérea. 2012;(2):96-111.

8. Amaral-Natalio M, Silva Nunes G, Herber V, Michaelsen SM Relação entre cadência da subida e descida de escada, recuperação motora e equilíbrio em indivíduos com hemiparesia. Acta Fisiátrica. 2011;18(3):146-50.

9. Oliveira LB, Cabral ACA, Holanda LJ, Neves MD, Rolim MM, Silva RGR. Efeitos da hidroterapia na hipertensão arterial sistêmica (HAS): uma revisão bibliográfica. Rev Científica Escola Saúde. 2013;2(2):65-75

10. Lisbôa AA, Silva Junior AC, Lima TB, Almeida RD. Efetividade da fisioterapia aquática no tratamento da dor lombar crônica: revisão sistemática com metanálises. Cad Grad Ciênc Biol Saúde. 2012;1(15):33-42.

11. Cruz DMC, Silva NS, Patti LP, Paiva G, Paolillo AR. Correlação entre sensibilidade, função manual e independência em indivíduos pósacidente vascular cerebral. Rev Para Med. 2015;29(1):23-30.

12. Schmitz FS, Stigger F. Atividades aquáticas em pacientes com paralisia cerebral: um olhar na perspectiva da fisioterapia. Rev Atenção Saúde. 2014;12(42):78-89.

http://dx.doi.org/10.13037/rbcs.vol12n42.2428
13. Foschiera AIC, Cartonilho G, Teles CBG. Prevalência da toxoplasmose em pacientes atendidos no laboratório centra de saúde pública de Porto Velho-RO. Saber Científico. 2009;2(1):92-103.

14. Araújo TM, Barros LM, Caetano JÁ, Moreira RAN, Frota MN, Feitosa ACL, et al. Neurotoxoplasmose boarding in patients with HIV/Aids in intensive care unit. J Nurs UFPE. 2012;6(5):1046-52. http://dx.doi.org/10.5205/reuol.2450-19397-1-LE.0605201212

15. Soares AV. A contribuição visual para o controle postural. Rev Neurocienc. 2010;18(3):370-379.

16. Moreira KLAF, Dornelas L, Tavares GR, Andrade SM. Intervenção fisioterapêutica e Síndrome da Imunodeficiência Adquirida associada à leucoencefalopatia e neurotoxoplasmose: relato de caso. Fisioter Mov. 2007;20(3):35-40.

17. Trindade APNT, Barboza MA, Oliveira FB, Borges APO. Influência da simetria e transferência de peso nos aspectos motores após Acidente Vascular Cerebral. Rev Neurociênc. 2011;19(1):61-7.

18 Krueger-Beck E, Scheeren EM, Nogueira-Neto GN, Button VLSN, Neves EB, Nohama P. Potencial de ação: do estímulo à adaptação neural. Fisioter Mov. 2011;24(3):535-47. http://dx.doi.org/10.1590/S0103-51502011000300018

19. Santos DG, Pegoraro ASN, Abrantes CV, Jakaitis F, Gusman S, Bifulco SC. Avaliação da mobilidade funcional do paciente com sequela de AVC após tratamento na piscina terapêutica, utilizando o teste Timed Up and Go. Einstein. 2011;9(3 Pt 1):302-6.

20. Avelar NCP, Bastone AC, Alcântara MA, Gomes WF. Efetividade do treinamento de resistência à fadiga dos músculos dos membros inferiores dentro e fora d'água no equilíbrio estático e dinâmico de idosos. Rev Bras Fisioter. 2010;14(3):229-36. http://dx.doi.org/10.1590/S1413-35552010000300007

21. Cruz IBM, Barreto DCM, Fronza AB, Jung IEC, Krewer CC, Rocha MIUM, etal. Equilíbrio dinâmico, estilo de vida e estados emocionais em adultos jovens. Braz J Otorhinolaryngol. 2010;76(3): 392-398. http://dx.doi.org/10.1590/S1808-86942010000300020

22. Meereis ECW, Favretto C, Souza J, Marques CLS, Gonçalves MP, Mota CB. Análise do equilíbrio dinâmico de idosas institucionalizadas após Hidrocinesioterapia. Rev Bras Geriatr Gerontol. 2013;16(1):41-47. http://dx.doi.org/10.1590/S1809-98232013000100005

23. Girondi JBR, Hammerschimidt KSA, Tristão FR, Fernandez DLR O uso do Índice de Barthel Modificado em idosos: contrapondo capacidade funcional, dependência e fragilidade. J Health Biol Sci. 2014:2(4):213-17.

http://dx.doi.org/10.12662/2317-3076jhbs.v2i4.106.p213-217.2014 